\title{
Spontaneous intraperitoneal rupture of the urinary bladder
}

\author{
LLOYD R. JENKINSON* \\ M.B., Ch.B.
}

Salford Royal Hospital, Salford M60 9EP

\begin{abstract}
Summary
Spontaneous rupture of the bladder is a rare occurrence but carries a high mortality. A female case is reported and the mode of presentation, investigation and management discussed
\end{abstract}

\section{Introduction}

Peritonitis due to spontaneous rupture of the bladder is a rare surgical emergency but has a mortality of up to $80 \%$ in undiagnosed cases (Thomson, Johnson and Ross, 1961). It should be considered in all patients with peritonitis of unknown aetiology and prompt investigation and surgery undertaken. The following case report illustrates the common mode of presentation but it is unusual in that the patient was female and it was due to squamous cell carcinoma of the bladder.

\section{Case report}

A 73-year-old woman was admitted with a 5-hr history of lower abdominal pain which had started suddenly in the right iliac fossa within minutes of defaecation. The pain spread rapidly to involve the whole of the lower abdomen, and increased in severity. There was associated nausea, vomiting and anorexia. Two weeks previously she had complained of dysuria and frequency which had been treated with antibiotics and her symptoms resolved. On examination she looked ill, was pale, perspiring and pyrexial. There was generalized abdominal tenderness with guarding, and bowel sounds were absent. Investigations showed a leucocytosis but there was no evidence of free gas on plain films of the abdomen. A diagnosis of perforated viscus was made and the patient prepared for laparotomy. Catheterization produced a small amount of cloudy urine.

At operation the peritoneal cavity contained one litre of cloudy fluid and there was a one-cm perforation in the fundus of the bladder through which urine was leaking. The perforation was enlarged and the bladder was found to contain an extensive tumour

\footnotetext{
*Present address: Orthopaedic Department, Royal Hallamshire Hospital, Glossop Road, Sheffield S10 2JF.
}

which was adherent to the uterus and had spread to the para-aortic nodes. A biopsy was taken and the bladder repaired with catgut. Peritoneal toilet was performed and the abdomen and bladder drained. Histology of the biopsy showed a poorly differentiated squamous cell carcinoma. Postoperatively the patient made a slow recovery. In the third week the blood urea began to rise and the patient's condition slowly deteriorated. She died 21 days after admission.

TABLE 1. Classification of spontaneous bladder rupture

Bladder wall lesions:

(1) Inflammatory
(a) Intravesical - Acute
- Chronic
- Tuberculous
- Schistosomiasis
- Following radiotherapy

(2) Neoplasia

(b) Extravesical

Obstructive:

(1) Bladder neck obstruction

(a) Intravesical - Calculus

- Papilloma

- Neurological diseases

(b) Extravesical - Prostatic

$$
\begin{aligned}
& \text { - Hypertrophy } \\
& \text { - Carcinoma } \\
& \text { - Uterine } \\
& \text { - Tumours } \\
& \text { - Pregnancy }
\end{aligned}
$$

(2) Urethral obstruction - Stricture

$$
\text { - Calculus }
$$$$
\text { - Tumour }
$$

Drugs: e.g. Carbachol

Idiopathic

\section{Discussion}

Most cases of intraperitoneal rupture of the bladder are associated with inflammation, neoplasia, or outlet obstruction and it is classified as shown in Table 1. It is more common in the male and it has been suggested that this is due to the anatomical 
features of the male urethra which increase the likelihood of bladder neck obstruction and hence distension with subsequent perforation (Glashan, 1967). Classically the patient presents with sudden onset of lower abdominal pain preceded by an increase in intra-abdominal pressure, for example, whilst micturating, straining at stool, retching, lifting, etc. The patient may also complain of an inability to micturate or may only be able to pass small volumes of urine. This may be blood-stained. In $10 \%$ of cases there is an indefinite history and also absence of abdominal pain (Bastable, DeJode and Warren, 1959). On examination there are signs of generalized or lower abdominal peritonitis but this is absent in those patients without abdominal pain.

On suspicion of the diagnosis the bladder should be catheterized and small volumes of infected or blood-stained urine may be obtained. The classical test, in which a measured volume of fluid is instilled into and then withdrawn and re-measured, does not obviate the need for cystography and may be omitted.

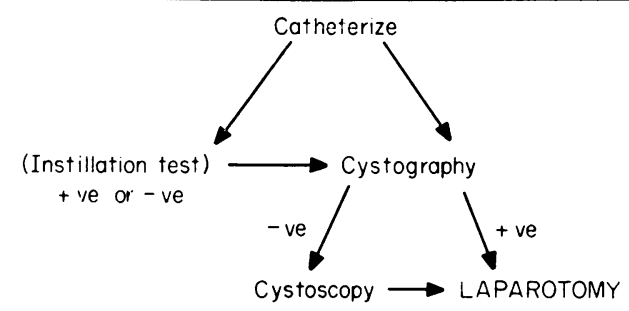

FIG. 1. Management.

Cystography should be performed in those patients whose condition permits and oblique, lateral and postmicturition films obtained. These will help in those patients with small perforations and will also indicate the site of rupture (Kalalis, 1971). In cases with normal cystograms, cystoscopy may provide the answer (Fig. 1).

On confirmation of the diagnosis laparotomy should be performed, a biopsy of the bladder wall carried out and the rupture repaired with one or two layers of catgut. The bladder and abdomen are then drained. In those cases where the diagnosis is not made pre-operatively, the cause of peritonitis may not be apparent and the site of leakage will only be $\stackrel{\mathcal{D}}{\rightarrow}$ demonstrated after distension of the bladder. The site and size of the rupture may indicate the underlying pathology. Large tears in the posterior paritoneal wall usually occur in the presence of a distended bladder (Oliver and Taguchi, 1964). This suggests a preceding bladder neck obstruction, for example, prostatic hypertrophy, and more definitive surgery may be required. Small perforations usually occur through bladder wall lesions.

This case demonstrates that failure to make the diagnosis pre-operatively is a common pit-fall; $\underline{3}$ however the cause of peritonitis was easily found. of It must be emphasized that a high index of suspicion should be maintained in any case of peritonitis of unknown cause, especially in those patients with a previous urological disorder, and the bladder should be distended and carefully inspected before the abdomen is closed and a diagnosis of idiopathic peritonitis made.

\section{Acknowledgments}

I would like to thank Mr J. R. N. Curt for permission to report this case of a patient under his care.

\section{References}

Bastable, J.G., DeJode, L.R. \& Warren, R.P. (1959) Spontaneous rupture of the bladder. British Journal of Urology, 31, 78.

GlashaN, R.W. (1967) Perforation as a complication of carcinoma of the bladder. British Journal of Urology, 39, 178 .

Kelalis, P.P. (1971) Trauma to the urinary system. In: Clinical Urography (Ed by Emmett, J.L. \& Witte), 3rd edn, p. 1734-1735. W. B. Saunders, Philadelphia.

Oliver, J.A. \& TAguchi, Y. (1964) Rupture of the full bladder. British Journal of Urology, 36, 524.

Thompson, I.M., Johnson, E.L. \& Ross, G. (1961) The acute abdomen of unrecognised bladder rupture. Archives of Surgery, 90, 371. 